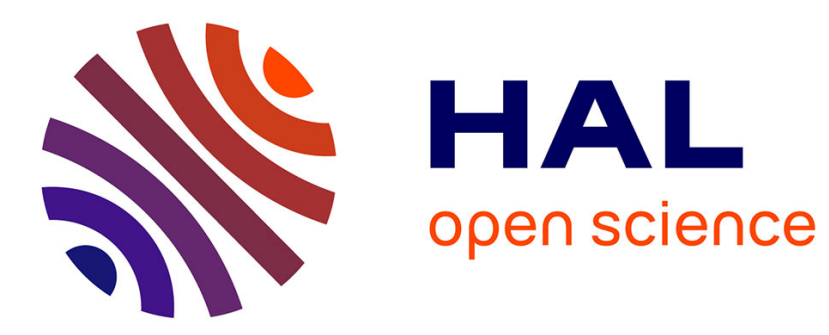

\title{
Experimental investigation of curved electrode actuator dynamics in viscous dielectric media
}

Sindhu Preetham Burugupally, David Hoelzle

\section{To cite this version:}

Sindhu Preetham Burugupally, David Hoelzle. Experimental investigation of curved electrode actuator dynamics in viscous dielectric media. Applied Physics Letters, 2018, 113 (7), pp.074102. 10.1063/1.5042456 . hal-02269939

\section{HAL Id: hal-02269939 \\ https://hal.science/hal-02269939}

Submitted on 23 Aug 2019

HAL is a multi-disciplinary open access archive for the deposit and dissemination of scientific research documents, whether they are published or not. The documents may come from teaching and research institutions in France or abroad, or from public or private research centers.
L'archive ouverte pluridisciplinaire HAL, est destinée au dépôt et à la diffusion de documents scientifiques de niveau recherche, publiés ou non, émanant des établissements d'enseignement et de recherche français ou étrangers, des laboratoires publics ou privés. 


\title{
Experimental investigation of curved electrode actuator dynamics in viscous dielectric media
}

\author{
Sindhu Preetham Burugupally ${ }^{1, \text { a) }}$ and David Hoelzle ${ }^{2}$ \\ 1) Department of Mechanical Engineering, Wichita State University, Wichita, KS 67260, \\ USA \\ ${ }^{2)}$ Department of Mechanical and Aerospace Engineering, The Ohio State University, Columbus, \\ OH 43210 USA
}

\begin{abstract}
Micromanipulation of biological cells inside a liquid environment requires an actuator that has a small footprint to reduce viscous drag, and low actuation voltage to prevent electrolysis and Joule heating. Curved electrode actuators hold the promise for underwater micromanipulation because they yield large displacements at low actuation voltages for a small footprint. In this letter, we report on the frequency-domain characteristics of the actuator and demonstrate that the actuator can achieve large displacements $(1-10 \mu \mathrm{m})$ and generates large forces $(1-21 \mu \mathrm{N})$ at low actuation voltages $(8 \mathrm{~V})$ over 1-1000 Hz frequency range in a viscous dielectric media.
\end{abstract}

Keywords: Curved electrode, dynamic response, electrostatic actuator, viscous dielectric media

An actuator designed to manipulate or probe biological cells at high speeds must be able to operate in an aqueous environment and achieve the following performance metrics: large displacement $(1-10 \mu \mathrm{m})$ and force $(1-25 \mu \mathrm{N})$; and broadband frequency $(1-1000 \mathrm{~Hz})$ at low actuation voltages $(\leq 8 \mathrm{~V}) .{ }^{1}$ Electrostatic actuators are shown to benefit from high relative permitivitty $\left(\epsilon_{r}=80\right)$ of aqueous media yielding large forces at low actuation voltages. ${ }^{2}$ However, in an aqueous environment, the viscous drag on the actuator surface decreases the quality $(Q)$ factor by at least four orders of magnitude, ${ }^{?}$ challenging large displacements at high frequencies. As such, comb drive actuators are not strong enough to achieve these metrics at the requisite voltages without large comb arrays which amplify viscous drag. Parallel plate actuators benefit from an electrostatic spring softening nonlinearity that enables high electrostatic force gains, and thus high speed operation, but this same nonlinearity limits displacement by the pull-in instability. ${ }^{3,4}$ Curved electrode actuators hold promise for large displacement, high bandwidth actuation in an underwater environment because of a mechanical spring hardening nonlinearity that counteracts the electrostatic spring softening nonlinearity, extending the usable displacement range while maintaining large electrostatic force gain from a small electrode gap..$^{5,6}$

Curved electrode actuators are designed to be operated at voltages, $V$, exceeding the pull-in voltage, $V_{P I}$ (Fig. 1). At $V>V_{P I}$, the beam electrode comes in contact with the curved electrode and increasing the voltage causes the contact point, $a$, to zip along the face of the curved electrode. This zipping action results in a shortening of the sprung beam length, $L-a$, leading to a spring hardening nonlinearity that counteracts the elec-

\footnotetext{
a)Electronic mail: sindhupreetham.burugupally@wichita.edu
}

trostatic spring softening nonlinearity, and thus a stable operation past the pull-in voltage, $V_{P I}$ (Fig. 1b). Additionally, the small contact distanced between the beam and curved electrodes provides high displacement gain, permitting large displacement $(10 \mu \mathrm{m})$, large force generation $(10 \mu \mathrm{N})$, and high frequency operation $(1000 \mathrm{~Hz})$ with low voltages $(8 \mathrm{~V})$ and small actuator footprints. To achieve the aforementioned performance metrics of the actuator, we redesigned a curved electrode actuator ${ }^{7-9}$ in a clamped-clamped beam configuration. ${ }^{5}$

In this letter, we investigate the dynamics of this actuator in a high viscous media, where $Q$-factor $\ll 1$. Here, we present experimental demonstration of the actuator dynamics in two different viscous media: deionized (DI) water and methanol, and demonstrate that the actuator can achieve the desired performance metrics, making it a suitable candidate for micromanipulation of biological cells suspended in aqueous media.

The actuator operates on the principle of electrostatics and is fabricated from silicon $(<100>$ p-type boron doped to $100 \mathrm{ppm}$ ) using micromachining techniques ${ }^{5}$ with the physical parameters given in Table I. The curved electrostatic actuator comprises of three main components: a beam electrode, a reinforcing beam, and a set of four curved electrodes (Fig. 1). The curved electrodes drive the beam electrode in forward and backward directions that deliver the generated force and displacement strokes to the reinforcing beam which in turn delivers to an external load. Note that the reinforcing beam increases the bandwidth frequency of the actuator. The curved electrodes are immovable rigid members whose contour $s(x)$ is described by a second order polynomial function, $s(x)=\delta_{m}(x / L)^{2} \forall x \in[0, L]$, where $\delta_{m}$ is the maximum throw of the curved electrode, $x$ is the coordinate along the beam electrode, and $L$ is the half-length of the beam electrode (Fig. 1b). The beam electrode and the reinforcing beam together form a compliant clamped- 
(a)
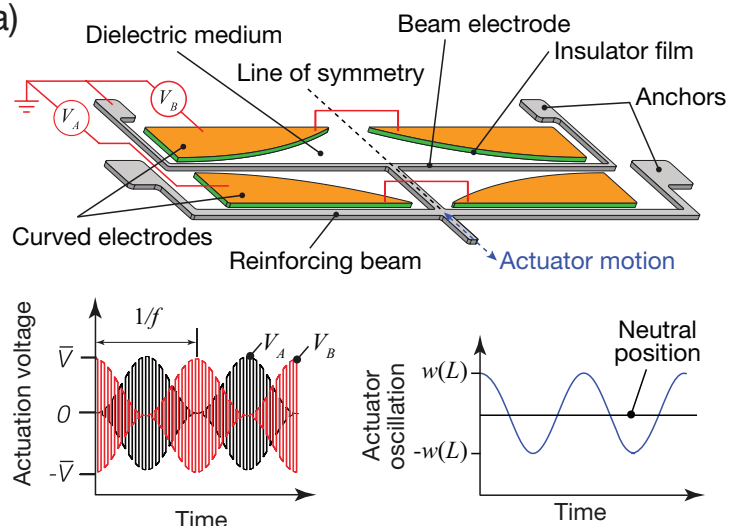

(b)

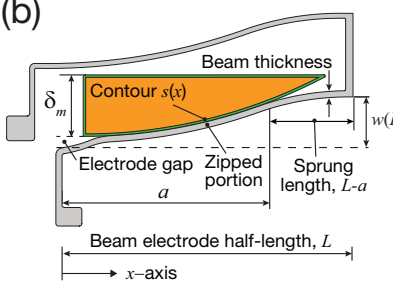

(c)

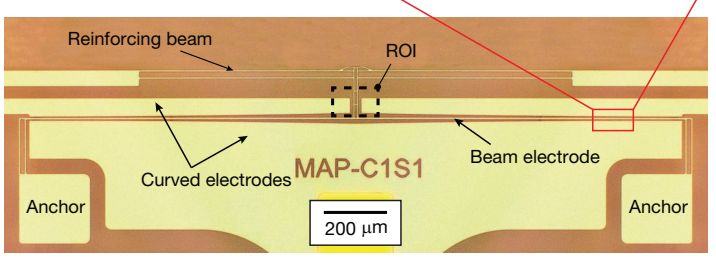

FIG. 1. Curved electrode actuator. (a) Schematic sketch of the actuator in unenergized state with actuation voltages $V_{A}$ and $V_{B}$, and actuator displacement $w(L)$ shown. (b) One-half of the actuator in zipped mode with annotated design parameters. (c) Stereomicroscopy of the top view of the actuator with the region of interest (ROI) shown by a black-dashed box. The figure inset shows a scanning electron microscopy (SEM) of the actuator electrode and its gaps (gap height = $30.7 \mu \mathrm{m}$; gap width $=3.8 \mu \mathrm{m}$ ) in side view after $65 \%$ completion of the DRIE BOSCH process. The silicon is yet to be etched to $45 \mu \mathrm{m}$ deep before released from glass substrate by HF wet etch (Reproduced with permission from Springer Nature Microsyst Technol (2018) 24: 3479. Copyright 2018 Springer-Verlag GmbH Germany, part of Springer Nature). See Ref. ${ }^{5}$ for fabrication details.

clamped structure. The curved electrodes and the beam electrode are coated with $10 \mathrm{~nm}$ thick aluminum oxide film for electrical insulation. The actuator is totally submerged in the viscous dielectric media such as DI water and methanol.

As shown in Fig. 1a, energizing the actuator by supplying two amplitude-modulated voltage signals $V_{A}(t)=$ $\frac{1}{2} \bar{V}(1+\cos 2 \pi f t) \Pi(t)$ and $V_{B}(t)=\frac{1}{2} \bar{V}(1-\cos 2 \pi f t) \Pi(t)$ will result in the shuttling of the actuator — back and forth in-plane - with a peak-to-peak displacement amplitude $2 w(L)$. Here, $\bar{V}$ is the voltage amplitude, $\cos 2 \pi f t$ and $-\cos 2 \pi f t$ are the modulation waveforms, $t$ is the time, $\Pi(t)=\frac{4}{\pi} \sum_{j=1}^{\infty} \frac{1}{2 j-1}(-1)^{j-1} \cos 2 \pi f_{c} j t$ is a high-
TABLE I. Nominal and measured actuator parameters, and physical parameters. Nominal parameters are given by a single number. The measured parameters are given as mean \pm standard deviation for four measurements.

\begin{tabular}{ll}
\hline \hline Parameter & Numerical value \\
\hline Beam electrode half-length, $L$ & $1000 \mu \mathrm{m}$ \\
Beam electrode height, $h$ & $45 \pm 0.4 \mu \mathrm{m}$ \\
Beam electrode thickness, $t$ & $4.7 \pm 0.2 \mu \mathrm{m}$ \\
Electrode gap, $g$ & $3.5 \pm 0.1 \mu \mathrm{m}$ \\
Reinforcing beam half-length, $l$ & $670 \mu \mathrm{m}$ \\
Reinforcing beam stiffness, $2 k_{r}$ & $4.4 \mathrm{~N} / \mathrm{m}$ \\
Dynamic viscosity of DI water & $0.89 \mathrm{mPa}-\mathrm{s}$ \\
Dynamic viscosity of methanol & $0.54 \mathrm{mPa}-\mathrm{s}$ \\
Viscous damping coefficient in DI water & $6.43 \mu \mathrm{N}-\mathrm{s} / \mathrm{m}$ \\
Viscous damping coefficient in methanol & $3.90 \mu \mathrm{N}-\mathrm{s} / \mathrm{m}$ \\
Young's modulus of $\mathrm{Si}^{10}, E$ & $130 \mathrm{GPa}$ \\
Relative permittivity of $\mathrm{Al}_{2} \mathrm{O}_{3}$ & 9 \\
Relative permittivity $\left(\epsilon_{r}\right)$ of DI water & 80 \\
Relative permittivity $\left(\epsilon_{r}\right)$ of methanol, & 32 \\
\hline
\end{tabular}

frequency, zero-mean pulse signal to prevent charge shielding effects ${ }^{11-13}$, and $f$ and $f_{c}=500 \mathrm{kHz}$ are the modulation (also called actuation) and carrier frequencies, respectively. The force $F$ generated by the actuator electrodes is estimated using the Newton's second law of motion equation, $F=2 k_{r} w(L)+b \dot{w}(L)+m_{e} \ddot{w}(L)$, where $2 k_{r}$ is the stiffness of the reinforcing beam, $w(L)$ is the displacement amplitude of the actuator or reinforcing beam, $b$ models the viscous damping on the reinforcing beam, $m_{e}$ is the effective mass of the reinforcing beam and inertial mass of the viscous media, and single overdot and double overdots are first and second temporal derivatives, respectively. The viscous damping coefficient $b$ is computed using the relation given in Shih et al. ${ }^{2} \mathrm{Be}-$ cause the reinforcing beam displacement is small in comparison to the available throw, we approximate damping coefficient $b$ to be a constant. The displacement and the force can be modulated by tuning the actuation voltages $V_{A}(t)$ and $V_{B}(t)$ (Fig. 1). Based on the actuation voltage amplitude $\bar{V}$ and the actuation frequency $f$, the actuator operates in one of the two modes: nonzipped and zipped. In the nonzipped mode, the beam electrode does not come in physical contact with the curved electrode, while in the zipped mode, the beam electrode comes in physical contact with the curved electrode (Fig. 1b).

The actuator is characterized in terms of actuator peak-to-peak displacement amplitude $2 w(L)$ at different voltage amplitudes $\bar{V} \in[4,8] \mathrm{V}$ in the increments of $1 \mathrm{~V}$ and actuation frequencies $f \in[1,4096] \mathrm{Hz}$ in the increments of $2^{N / 2}$, where $N \in[0,24]$. The actuator is taped to the bottom of a petri dish and the petri dish is filled with the viscous media of interest. The test facility is comprised of an inverted microscope (Zeiss Observer A1 microscope, Oberkochen, Germany) and high-speed camera (Miro M110, Vision Research, Wayne, NJ, USA) for cinephotomicrography of the moving actuator in the region of interest ROI (Fig. 1c), LabVIEW/NI PCIe-6343 data acquisition system for triggering the high-speed 
(a)

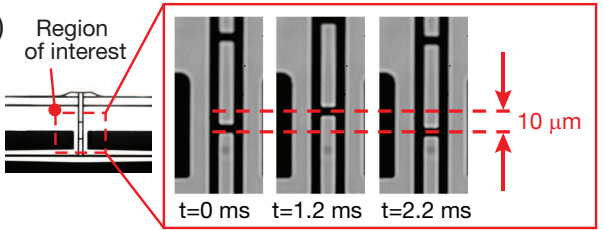

(b)

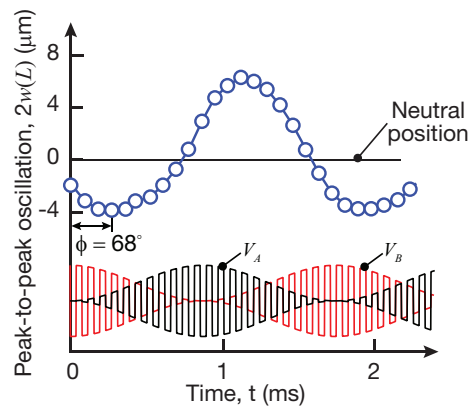

FIG. 2. (a) Time-lapsed images of one period of oscillation in the region of interest (shown in the red-dashed box). (b) Measured displacement trace of the actuator in deionized water for $8 \mathrm{~V}$ at $512 \mathrm{~Hz}$ in the region of interest (ROI). The displacement $2 w(L)$ is shown in blue solid line, while the actuation voltages $V_{A}$ and $V_{B}$ are shown in black and red solid lines, respectively. When no voltage is applied, the actuator is in the neutral position. The displacement measurement has a quantization error of $\pm 1 / 2$ pixel $= \pm 0.15 \mu \mathrm{m}$.

camera to initiate cinephotomicrography and generating two modulation waveforms $V_{a}(t)=\frac{1}{2} \bar{V}(1+\cos 2 \pi f t)$, $V_{b}(t)=\frac{1}{2} \bar{V}(1-\cos 2 \pi f t)$, a function generator (Tektronix AFG3022C, Beaverton, OR, USA) to multiply these modulation waveforms by $\Pi(t)$ and to generate two amplitude-modulated voltage waveforms $V_{A}$ and $V_{B}$, and three $\mathrm{x}-\mathrm{y}-\mathrm{z}$ test probes (XYZ Micropositioner, Quater Research and Development, Bend, OR, USA) to connect the ground and voltages $V_{A}$ and $V_{B}$ to the actuator contact pads. For given actuation voltages $V_{A}$ and $V_{B}$, the cinephotomicrography was recorded at frame rates $>10$ times the corresponding actuation frequency $f$; however, for $f=4096 \mathrm{~Hz}$, the frame rate is set to $6.1 f$ due to the light sensitivity limits of our high-speed camera.

The actuator is first characterized in deionized water and later in methanol media. During the change of media, the actuator is first triple rinsed in the later media (methanol) and then three times flushed with media and cyclically driven to full peak-to-peak displacement amplitude for 10 minutes. A custom image processing MATLAB $^{\circledR}$ script is used to segment the cinephotomicrograph into frames, measures the actuator location in each frame, and then creates time traces of this measurement for follow-up analyses of frequency content and peak-to-peak displacement amplitude $2 w(L)$.

The actuator displacement is measured in the region of interest (ROI) using the high-speed camera and inverted microscope (Fig. 1c). Fig. 2 provides a representative displacement signal trace at high-frequency operation in deionized water. Note that at this frequency the dis-

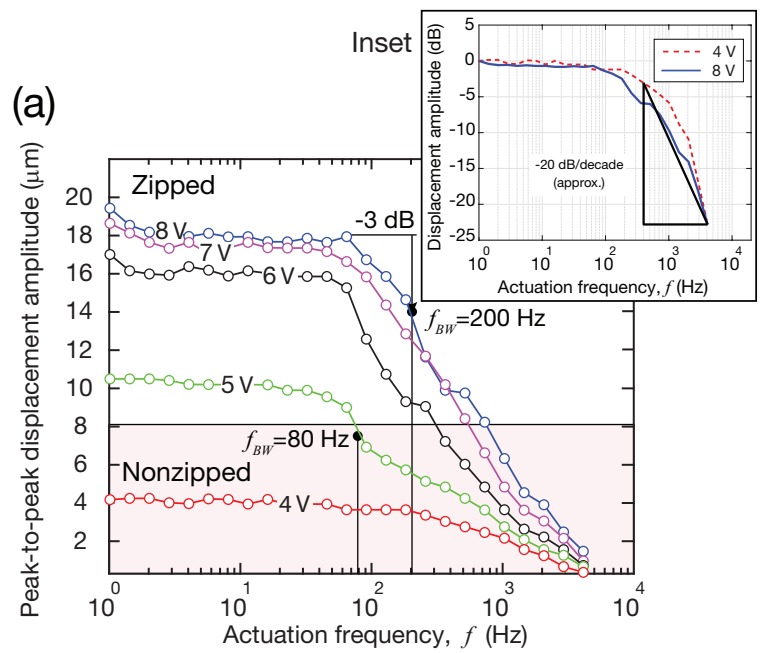

(b)

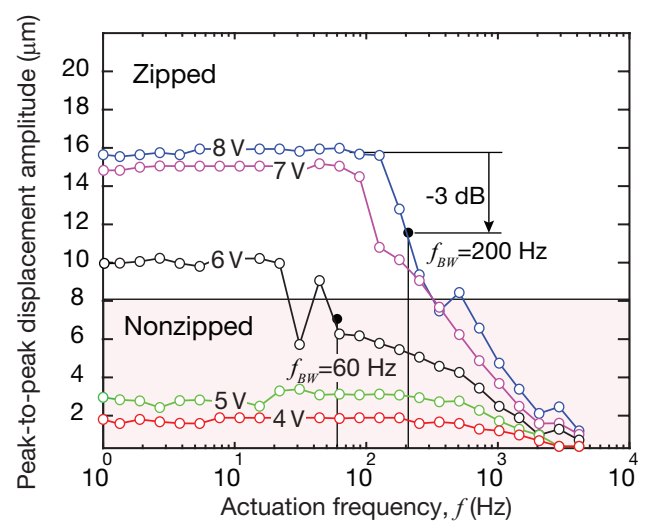

FIG. 3. Measured frequency response of the actuator for different actuation voltage amplitudes $\bar{V} \in[4,8] \mathrm{V}$ in (a) deionized water (b) methanol. In figure (a) inset, the displacement amplitude defined in terms of decibels $(\mathrm{dB})$ is scaled with respect to the actuator displacement amplitude $w(L)$ at frequency $f=1 \mathrm{~Hz}$ and corresponding actuation voltage amplitude $\bar{V}=4$ or $8 \mathrm{~V}$.

placement trace approximates a sinusoidal wave and that the peak displacement lags the actuation signal peak $V_{B}$. Actuator performance over the entire operating range is better elucidated in frequency response plots in Fig. 3. The most obvious performance features is that the actuator operates in two basic modes - nonzipped and zipped. Additionally, the actuator behaves as a first-order overdamped system, as characterized by the $-20 \mathrm{~dB} /$ decade magnitude slope at high frequencies (Fig. 3a inset). Our $Q$-factor is $\ll 1$ because of the highly viscous environment; the lower frequency of the two real poles is at approximately $200 \mathrm{~Hz}$ and the higher frequency real pole is beyond the frequency range we tested. There are more subtle features of the input voltage amplitude-frequency operating space that will be described in the following.

1. Beam electrode zipping greatly enhances the actuator displacement, at least doubling the displacement (Fig. 3). In the zipped mode, the beam 

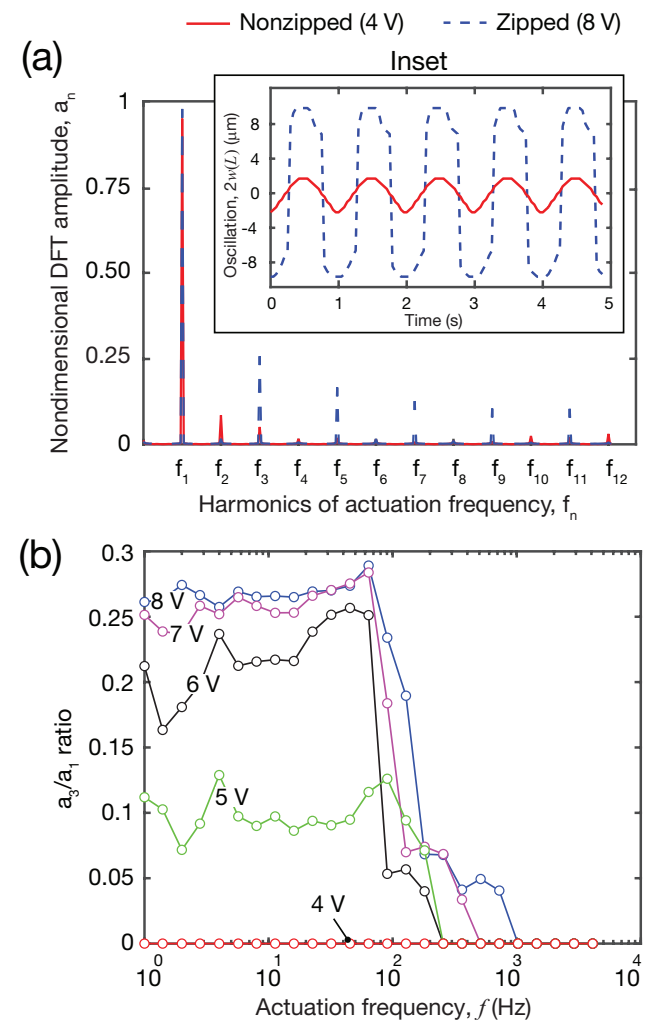

FIG. 4. Measured actuator response in deionized water. (a) Discrete Fourier transform (DFT) of the measured actuator displacement at $f=1 \mathrm{~Hz}$ actuation frequency in zipped and nonzipped operations. Here, the frame rate is 40 times the actuation frequency $f$ and hence prevents aliasing for harmonic frequencies of up to $19^{\text {th }}$ order, $f_{19}$. The nondimensional DFT amplitude $a_{n}$ is scaled with actuator peak-to-peak amplitude $\max 2 w(L)$ as shown in equation 1. Figure inset shows actuator displacement trace for nonzipped $(4 \mathrm{~V})$ and zipped $(8 \mathrm{~V})$ at $1 \mathrm{~Hz}$. The displacement measurement has a quantization error of $\pm 1 / 2$ pixel $= \pm 0.15 \mu \mathrm{m}$. (b) Ratio of DFT amplitudes $a_{3} / a_{1}$ over $1-4096 \mathrm{~Hz}$ frequency range for different voltages, where $a_{1}$ and $a_{3}$ correspond to DFT amplitudes at frequencies $f_{1}$ and $f_{3}$, respectively.

and curved electrodes of the actuator will come in physical contact, greatly reducing the electrode gap and providing a strong amplification of electrostatic force, hence a higher actuator displacement $w(L)$. A high relative permitivitty of the media also enhances the actuator displacement. For instance, in low actuation frequencies $f<100 \mathrm{~Hz}$ where the inertial and viscous fluid effects on the actuator are negligible - it can be seen that the actuator's displacement $2 w(L)$ is enhanced in deionized water (high $\epsilon_{r} ; \epsilon_{r}=80$ ) by up to $6 \mu \mathrm{m}$ and $2 \mu \mathrm{m}$ in zipped and nonzipped modes, respectively, relative to methanol (low $\epsilon_{r} ; \epsilon_{r}=32$ ) (Fig. 3). The difference in force generated by the actuator, hence displacement, would be even higher in comparison to actuation in air (low $\epsilon_{r} ; \epsilon_{r}=1$ ), as has been demonstrated by Legtenberg et al. ${ }^{7}$
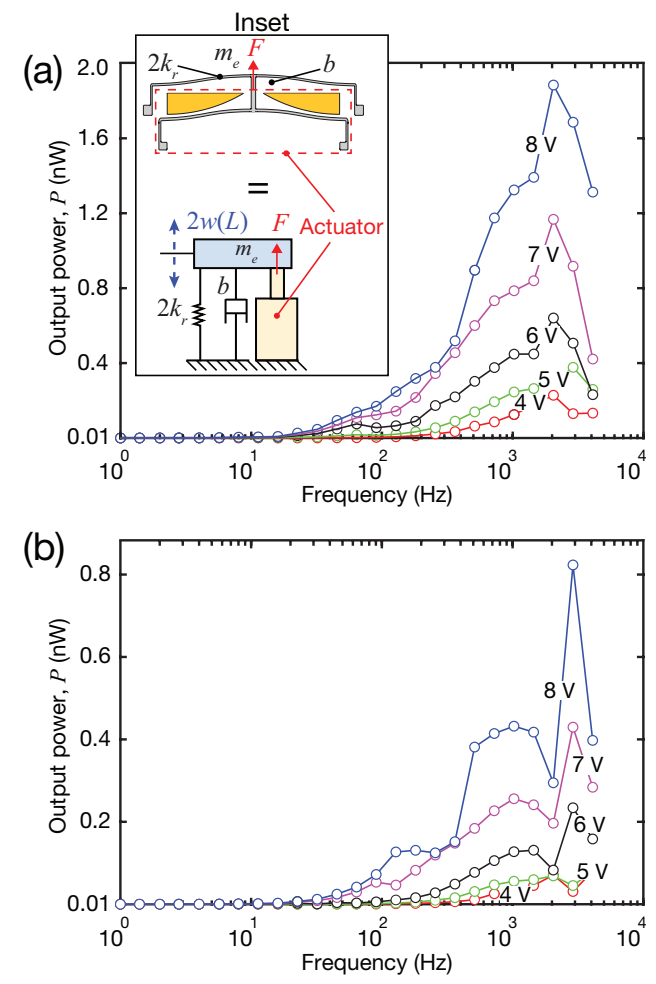

FIG. 5. Estimated available power at the reinforcing beam of the actuator over 1-4096 Hz frequency range for different actuation voltage amplitudes $\bar{V} \in[4,8] \mathrm{V}$ in (a) deionized water (b) methanol. Inset shows a schematic sketch of the actuator and its equivalent lumped model with stiffness $2 k_{r}$, damping coefficient $b$, effective mass $m_{e}$, and generated force $F$ applied on the reinforcing beam.

2. Zipping enhances a system nonlinearity that causes an increase in bandwidth frequency with increasing $\bar{V}$ (Fig. 3). For instance, the actuator bandwidth frequencies $f_{B W}$ in deionized water for $5 \mathrm{~V}$ and $8 \mathrm{~V}$ are approximately 80 and $200 \mathrm{~Hz}$, respectively. Similarly, the bandwidth frequencies $f_{B W}$ in methanol media for $6 \mathrm{~V}$ and $8 \mathrm{~V}$ are approximately 60 and $200 \mathrm{~Hz}$, respectively. In the zipped operations, the effective stiffness of the actuator $k$ is proportional to $\frac{1}{(L-a)^{3}}$, where $L-a$ is the sprung length of the electrode. A higher the voltage amplitude $\bar{V}$ will result in a shorter sprung length and higher actuator stiffness, and thus a higher bandwidth frequency. On the contrary, the media introduces inertial loading and viscous damping, both of which are known to decrease the bandwidth frequency and increase actuator pull-in times.

3. Although the actuator is a nonlinear system ${ }^{5}$, the actuator dynamics are approximately linear in the nonzipped mode: low $\bar{V}$ and/or high $f$ (Fig. 4). Applying a sinusoidal forcing function with frequency $f=1 \mathrm{~Hz}$ on the actuator for the zipped mode $(8 \mathrm{~V})$ excites the higher odd harmonics $f_{2 n+1}$ $\left(\forall n \in \mathbb{Z}_{>0}\right)$ in the actuator, as measured by ap- 
plying the discrete Fourier transform (DFT) to the displacement trace $2 w(L)$. Note that the $n^{\text {th }}$ order harmonic frequency $f_{n}=n f$ and the nondimensional DFT amplitude

$$
a_{n}=\frac{\frac{2}{M}\left|\sum_{m=0}^{M-1} 2 w(L)_{\mid m} e^{-j \frac{2 \pi n m}{M}}\right|}{\max 2 w(L)_{\mid f=1 \mathrm{~Hz}}} .
$$

As can be seen in equation $1, a_{n}$ is scaled with peak-to-peak actuator amplitude, $\max 2 w(L)$ for $f=1 \mathrm{~Hz}$. Here, $j=\sqrt{-1}$ and $M$ is the number of sample points used in displacement measurement $2 w(L)$. The presence of higher odd harmonics in the zipped mode results in a clipped sinusoidal response instead of an approximately sinusoidal response. This bears a resemblance of the characteristics of a quasi-harmonic nonlinear system with an external harmonic force of frequency $f_{1} \cdot{ }^{14}$ However, for the nonzipped mode $(4 \mathrm{~V})$, the higher odd harmonics $f_{2 n+1}(\forall n \in \mathbb{N})$ vanish with the appearance of a very low amplitude $\left(<0.1 a_{1}\right)$ second harmonic response $f_{2}$. In this operating mode, the actuator approximates a linear system. In the high $\bar{V}$ and high $f$ operation, the actuator is again nonzipped, yielding a $f_{3}$ harmonic that vanishes in relation to the $f_{1}$ harmonic (Fig. 4c). Please note that for a high actuation frequency $f=4096 \mathrm{~Hz}$, the frame rate is set to $6.1 f$ due to the limits placed by the camera speed (frames per second); here, the acquisition of the actuator displacement signal $2 w(L)$ containing the third harmonics $\left(f_{3}\right)$ satisfies the Nyquist-Shannon Sampling Criterion $\left(\geq 2 f_{3}\right)$, but the sampling rate is below the commonly accepted rule-of-thumb of 10 times the frequency of interest.

4. The available mechanical power $P$ at the reinforcing beam of the actuator is on the order of a nano-Watt, calculated using the relation $P=$ $\frac{1}{f} \int_{t=0}^{1 / f} \int_{x=0}^{2 l} F . \mathrm{d} w(x, t)$ (Fig. 5). The reinforcing beam displacement $w(x, t)$ is given by the relation $w(x, t)=\phi(x) w(L, t)$, where $\phi(x)$ is the approximate first mode shape for a clamped-clamped beam ${ }^{15}$ and $w(L, t)$ is the displacement of actuator at $x=L$ (equals displacement of the reinforcing beam at $x=l$ ). Note that the work done by the beam sprung force and inertia force over one oscillation are zero. Since there is no external load on the reinforcing beam, the available mechanical power is dissipated as heat due to the viscous effect of the media. The available power peaks at $2048 \mathrm{~Hz}$ and $2896 \mathrm{~Hz}$ for deionized water and methanol, respectively. The sudden dip at $2048 \mathrm{~Hz}$ in methanol is due to the smaller actuator displacement $2 w(L)$ at $2048 \mathrm{~Hz}(2.0 \mu \mathrm{m})$ compared to $2896 \mathrm{~Hz}(2.4 \mu \mathrm{m})$ (Fig. 3).

In summary, this letter details an experimental characterization of the dynamics of a curved electrode actuator, which has the favorable properties of a large displacement and high frequency range with a low input voltage, but has not been sufficiently described for dynamic operation in viscous media. Four experimental observations are reported: (1) a higher relative permittivity of the media has enhanced the actuator displacement; (2) the zipping mode of operation has enhanced the operating range of the actuator in terms of displacement and bandwidth frequency; (3) to analytically describe the actuator motion, a minimum of first six odd normal modes are required; and (4) the mechanical power available at the reinforcing beam is on the order of a nano-Watt. The experiment results: provide an indication of how many normal modes will be required to analytically describe motion in future theoretical and computational studies; and demonstrate that the actuator can achieve displacements on the order of a biological cell diameter $(1-10 \mu \mathrm{m})$ and generates large forces $(1-21 \mu \mathrm{N})$ at low actuation voltages $(8 \mathrm{~V})$ over $1-1000 \mathrm{~Hz}$ frequency range in a viscous aqueous media, making the actuator a suitable microsystem for micromanipulation of biological cells suspended in aqueous media.

This work was supported in part by University of Notre Dame Walther Cancer Foundation IITP Fellowship, American Cancer Society Award IRG 14-195-01, Notre Dame Clare Boothe Luce Fellowship, Notre Dame Advanced Diagnostics \& Therapeutics Berry Family Foundation Fellowship, and Wichita State, Notre Dame, and Ohio State capitalization funds. Technical consultation was given by the Notre Dame Nanofabrication Facility.

${ }^{1}$ B. S. Preetham, M. A. Lake, and D. J. Hoelzle, Napa Microsystems Workshop 2017 (2017).

${ }^{2}$ R. M. Shih, D. S. Contreras, T. L. Massey, J. T. Greenspun, and K. S. J. Pister, in 2018 IEEE Micro Electro Mechanical Systems (MEMS) (2018) pp. 596-599.

${ }^{3}$ S. Warnat, H. King, C. Forbrigger, and T. Hubbard, Journal of Micromechanics and Microengineering 25, 025011 (2015).

${ }^{4}$ S. D. Senturia, Microsystem Design (Springer US, 2001).

${ }^{5}$ B. S. Preetham, M. A. Lake, and D. J. Hoelzle, Journal of Micromechanics and Microengineering 27, 095009 (2017).

${ }^{6}$ S. P. Burugupally and J. A. Mangels, Microsystem Technologies (2018), 10.1007/s00542-018-3751-3.

${ }^{7}$ R. Legtenberg, J. Gilbert, S. D. Senturia, and M. Elwenspoek, Journal of Microelectromechanical Systems 6, 257 (1997).

${ }^{8}$ P. M. Osterberg and S. D. Senturia, Journal of Microelectromechanical Systems 6, 107 (1997).

${ }^{9}$ J. Li, M. P. Brenner, T. Christen, M. S. Kotilainen, J. H. Lang, and A. H. Slocum, Journal of Microelectromechanical Systems 14, 1283 (2005).

${ }^{10}$ M. A. Hopcroft, W. D. Nix, and T. W. Kenny, Journal of Microelectromechanical Systems 19, 229 (2010).

${ }^{11}$ D. J. Hoelzle, C. K. Chan, M. B. Scott, M. A. Lake, and A. C. Rowat, Journal of Applied Physics 117, 014503 (2015), http://dx.doi.org/10.1063/1.4905385.

${ }^{12}$ V. Mukundan and B. L. Pruitt, Journal of Microelectromechanical Systems 18, 405 (2009).

${ }^{13}$ T. L. Sounart, T. A. Michalske, and K. R. Zavadil, Journal of Microelectromechanical Systems 14, 125 (2005).

${ }^{14}$ L. Meirovitch, Fundamentals of Vibrations (Waveland Press, Inc, 2001). 
${ }^{15}$ M. Sathyamoorthy, Nonlinear Analysis of Structures (1997), $\quad$ CRC Press Revivals (CRC Press, 2017). 